\title{
Bulk and electrochemical properties of $\mathrm{BiVO}_{4}$
}

\author{
I.C. Vinke, J. Diepgrond, B.A. Boukamp, K.J. de Vries ${ }^{1}$ and A.J. Burggraaf \\ Laboratory for Inorganic Chemistry, Materials Science and Catalysis, Department of Chemical Technology, University of Twente, \\ P.O. Box 217, 7500 AE Enschede, The Netherlands
}

Received 21 May 1991; accepted for publication 9 March 1992

\begin{abstract}
The mixed (oxygen ionic-electronic) conductor bismuth vanadate $\left(\mathrm{BiVO}_{4}\right)$ was studied with respect to its electrochemical properties. The ionic transference numbers, measured by the concentration cell method in the temperature range of 800 to 1000 $\mathrm{K}$, vary from 0.7 to 0.3 . The total conductivity of this ceramic material is one order of magnitude lower than found for cubic yttria-stabilized zirconia (YSZ). The activation enthalpy for the electronic conductivity is high ( $193 \mathrm{~kJ} / \mathrm{mol}$ ) compared to the ionic conductivity $\left(71 \mathrm{~kJ} / \mathrm{mol}\right.$ ). The $P_{\mathrm{O}_{2}}$ dependency of the conductivity data in combination with the Seebeck measurements showed electrons to be the majority charge carriers, indicating that $\mathrm{BiVO}_{4}$ is an $n$-type mixed conductor.
\end{abstract}

\section{Introduction}

In solid oxide fuel cells and comparable solid state ionic devices, the cathodic reaction often accounts for high energy losses. Therefore a continuing search for better cathode materials is performed world wide.

In the cathodic reaction on oxygen ion conducting materials, generally the adsorption of oxygen plays an important role. As oxygen is an electrophilic species, an electron rich surface may promote the adsorption and dissociation of oxygen. An $n$-type mixed conductor will supply such a high concentration of electrons. For reasons of compatibility with the electrolyte material to be used (erbia-stabilized bismuth oxide) a bismuth containing mixed conducting oxide was selected. Moreover, there are indications that bismuth is catalytically active in the oxygen oxidation/reduction reaction [1].

In literature the only bismuth containing mixed conducting oxide of which $n$-type electronic conductivity is reported, is the solid solution of vanadium oxide and bismuth oxide. Although the literature on $\mathrm{BiVO}_{4}$ is extensive because of the use of this compound as a pigment, electrochemical data concerning this system are scarce. The information that can be found is highly contradictory. Ramadass et al.

\footnotetext{
1 Author to whom all correspondence should be addressed.
}

[2] reported that, depending on the stoichiometry, $\mathrm{BiVO}_{4-x}$ showed $p$-type or $n$-type mixed conductivity. Lu et al. [3] reported $\mathrm{BiVO}_{4}$ to be a p-type mixed conductor. Neither of these papers provided conclusive evidence for the type of electronic conductivity. Preliminary experiments indicated that $\mathrm{BiVO}_{4}$ might be an interesting material $[4,5]$.

For this reason a research program was started in which the crystal structure, the stability, the electrical and ionic conductivity and the electrode properties of $\mathrm{BiVO}_{4}$ were studied. Also a comparison of a number of methods for the synthesis of $\mathrm{BiVO}_{4}$ has been made.

\section{Experimental procedures}

\subsection{Synthesis}

Three methods for the synthesis of $\mathrm{BiVO}_{4}$ were tested: (i) Co-precipitation, (ii) solid state reaction, (iii) pyrolysis. The co-precipitation method turned out to be unsuccessful as the vanadyl ions are amphoteric, i.e. they dissolve in acid as well as in basic solutions. This means that co-precipitation of vanadium by changing the $p \mathrm{H}$ of a stoichiometric solution of the metal ions is difficult.

The solid state reaction (denoted SSR) was per- 
formed by mixing stoichiometric amounts of high purity grade $\mathrm{Bi}_{2} \mathrm{O}_{3}$ and $\mathrm{V}_{2} \mathrm{O}_{5}$ (Both from Merck). The oxides were mixed in isopropanol in a planetary mill (Pulvrisette 5) for $16 \mathrm{~h}$. After drying for $12 \mathrm{~h}$ at $400 \mathrm{~K}$ the obtained powder was ball-milled (Pulvrisette 0 ) for $45 \mathrm{~min}$ and calcined for $16 \mathrm{~h}$ at 1073 $\mathrm{K}$. This latter temperature was selected from DTA experiments of a mixture of $\mathrm{Bi}_{2} \mathrm{O}_{3}$ and $\mathrm{V}_{2} \mathrm{O}_{5}$ and lies well above the temperature of $913 \mathrm{~K}$ at which the reaction peak was observed in the DTA recording.

The pyrolysis method was performed by slightly modifying the method of Kruidhof et al. [6]. Stoichiometric amounts of $\mathrm{NH}_{4} \mathrm{VO}_{3}$ and $\mathrm{Bi}_{2} \mathrm{O}_{3}$ were dissolved in diluted and in concentrated nitric acid respectively. Citric-acid was added to the solutions in a ratio of $3 \mathrm{~mol}$ citric-acid per mol of metal ions dissolved. The solutions were mixed and diluted three times with distilled water. This solution was neutralized with ammonia ( $7 \mathrm{M}$ ). Precipitation that may occur will dissolve by adding an excess of ammonia. The basic solution was heated in small portions on a hot plate until the water had evaporated and the residue ignited. After the pyrolysis the powder of the mixed oxides was calcined during five hours at 1073 K.

Because of the widespread use of $\mathrm{BiVO}_{4}$ as a pigment it is commercially available (CERAC. 200 mesh ). For comparison reasons this powder was also used in a number of experiments.

The composition and crystal structure of the calcined powders was analyzed using XRD and DTA. The stability of the powder was studied using DTA.

\subsection{Ceramic sample preparation}

Ceramic samples of the different powders were prepared by milling the oxides in acetone for four hours. After drying the powder was pressed uniaxially into boules with a diameter of $14 \mathrm{~mm}$ and subsequently isostatically pressed $\left(7 \times 10^{10} \mathrm{~N}\right)$. The pressed boules were then sintered for $16 \mathrm{~h}$ at 1153 $\mathrm{K}$ in their own atmosphere, obtained by placing the boules on a layer of $\mathrm{BiVO}_{4}$ powder, to prevent selective evaporation of one of the metal oxides. The furnace was heated and cooled with 1 degree per minute.

The boules were cut to discs with a diameter of 12 $\mathrm{mm}$ and $2 \mathrm{~mm}$ thickness. These discs were ground and polished on both sides. For the final polish $6 \mu \mathrm{m}$ diamond paste was used. After polishing the samples were cleaned ultrasonically and annealed for one hour at $1023 \mathrm{~K}$ (heating and cooling rate as before).

When necessary for the experiments $300 \mathrm{~nm}$ thick gold electrodes were sputtered on the samples by dcsputtering.

\subsection{Electrochemical measurements}

On samples from both synthesis methods, as well as on samples from the commercial powder, ionic transference number $\left(t_{\mathrm{i}}\right)$ measurements were performed using the concentration cell technique [7]. In this technique a gas-tight sample of the material to be investigated was used to separate two compartments containing different oxygen partial pressures. The potential difference between two circular porous gold electrodes deposited on the opposite faces of the disk shaped sample was measured. The ionic transference number $t_{\mathrm{i}}$ was obtained by dividing the measured potential difference over the sample by the potential calculated from the Nernst equation.

The ionic transference number measurements were performed at several temperatures. The $P_{\mathrm{O}_{2}}$ value on one side of the sample was maintained at $1.0 \mathrm{~atm}$ and at $0.21 \mathrm{~atm}$ on the other side.

For checking the performance of the measurement cell, at regular intervals measurements with a pure ionic conductor (YSZ) were done.

Two-electrode conductivity experiments were performed by impedance spectroscopy. The measurements were carried out at several temperatures and oxygen partial pressures using circular porous gold electrodes. The impedance data were analyzed using the impedance data analysis program "Equivalent Circuit" [8].

The ionic and electronic conductivities were calculated from the total conductivity using the ionic transference numbers and a NLLS-fit procedure that is discussed below.

Thermal EMF experiments were performed to verify the type of electronic charge carriers in $\mathrm{BiVO}_{4}$ according to the method described by Wagner [9] The experiments were conducted on a cylindrical sample with a diameter and a length of $6 \mathrm{~mm}$. Gold contacts were sputtered on both ends of the cylinder. 
The Seebeck coefficient is defined as $\lim _{\Delta T \rightarrow 0}(-\Delta V /$ $\Delta T)$. Four different temperature gradients were applied across the sample and the resulting EMF values were recorded. The Seebeck coefficient was calculated from the slope of the straight line through the $\Delta T / E M F$ pairs. This eliminates a possible Soret effect.

\section{Results and discussion}

\subsection{Characterization}

All powders obtained from the different synthesis routes were analyzed by XRD. The diffractograms of the latter powders all show a single monoclinic crystal phase. No additional peaks were observed indicating that no free $\mathrm{Bi}_{2} \mathrm{O}_{3}$ or $\mathrm{V}_{2} \mathrm{O}_{5}$ was present. The cell parameters for the different powders were within the experimental errors. According to powder diffraction file (PDF No. 14-688) this should be a monoclinic phase with the space group I1 2/A 1 . Comparison of the measured data and the data obtained from the PDF in table 1 shows a good agreement of the $a$-axis. The $b$-and $c$-axis are $0.06 \AA$ shorter than reported in the PDF. This difference can be attributed to the precision of the floating point numbers used in the analysis program in calculating the cell parameters.

DTA experiments in air in the temperature range 573-1073 $\mathrm{K}$ show that no phase changes occur. Hence the monoclinic phase is stable in this temperature range. No peak was observed at the melting temperature of $\mathrm{V}_{2} \mathrm{O}_{5}(963 \mathrm{~K})$ indicating that virtually no free $\mathrm{V}_{2} \mathrm{O}_{5}$ was present in the sample. This is in agreement with the observed single phase $\mathrm{X}$-ray diagram. The melting temperatures of $\mathrm{BiVO}_{4}(1213$

Table 1

Monoclinic cell parameters for $\mathrm{BiVO}_{4}$. Powder diffraction file (PDF) values are given for reference purpose.

\begin{tabular}{lcc}
\hline Axis $(\AA)$ & This work & PDF \\
\hline$a$ & 5.195 & 5.195 \\
$b$ & 11.692 & 11.701 \\
$c$ & 5.086 & 5.092 \\
$\beta$ & 98.65 & 98.38 \\
$\rho_{\mathrm{th}}\left(\mathrm{g} / \mathrm{cm}^{3}\right)$ & 6.966 & 6.949 \\
\hline
\end{tabular}

$\mathrm{K})$ and $\mathrm{Bi}_{2} \mathrm{O}_{3}(1133 \mathrm{~K})$ are outside the temperature range of the DTA apparatus and therefore cannot be observed.

\subsection{Ceramic sample preparation}

Ceramic samples were prepared from all three powders (solid state reaction, pyrolysis and commercial powder). The densities of the boules after isostatic pressing and after sintering are presented in table 2 . The highest final relative density $\left(\rho_{\text {rel }}\right)$ obtained is approximately $92 \%$ and is observed for the sample from the solid state reaction method. This is equal to the value reported by Lu et al. [2]. The colour of the boules after sintering is purple-brown. We observed that if the sample from the pyrolysis method was sintered again at a temperature of $1153 \mathrm{~K}$ the relative density increases to $94.3 \%$. Increasing the sinter temperature to $1203 \mathrm{~K}$ resulted in a decrease of the $\rho_{\text {rel }}$ to $91 \%$. After sintering at this temperature, bubbles were found on the surface of the boule.

Removing the outer layer from the boules revealed an inhomogeneously coloured sample. The matrix of the sample is dark yellow with a large number of purple-brown spots. Electron microscopic investigations showed that the dark spots consists of material with a higher density that the surrounding matrix. EDS analysis of the matrix and the dark spots showed no difference in composition, i.e. no noticeable phase separation occurs.

The decrease in density with increasing temperature, the colour of the outer layer of the boules and the inhomogeneous density distribution in the bulk of the samples leads to the conclusion that $\mathrm{BiVO}_{4}$ rapidly forms a gas tight skin during sintering. This skin prohibits the escape of gases trapped in the bulk of the boules. Repeated sintering at higher temperatures may cause inflation of the sample causing a

Table 2

Densities of the samples before (1) and after (2) sintering at the given temperature.

\begin{tabular}{lclll}
\hline $\begin{array}{l}\text { Synthesis } \\
\text { route }\end{array}$ & $\begin{array}{l}\rho_{\text {rel }}^{(1)} \\
(\%)\end{array}$ & $\begin{array}{l}T_{\mathrm{s}} \\
(\mathrm{K})\end{array}$ & $\begin{array}{l}\rho_{\text {abs }} \\
\left(\mathrm{g} / \mathrm{cm}^{3}\right)\end{array}$ & $\begin{array}{l}\rho_{\text {rel }}^{(2)} \\
(\%)\end{array}$ \\
\hline pyrolysis & 82.5 & 1073 & 6.102 & 87.6 \\
SSR & 77.7 & 1103 & 6.430 & 92.3 \\
commercial & - & 1133 & 6.088 & 87.4 \\
\hline
\end{tabular}


decrease in density as was observed. This trapping of gas can also explain the formation of bubbles on the surface of the boules.

\subsection{Electrochemical measurements}

The results of the ionic transference number measurements of $\mathrm{BiVO}_{4}$ are presented in fig. 1 together with the reference measurements on YSZ. The data for YSZ are close to unity as is expected. The ionic transference numbers for $\mathrm{BiVO}_{4}$ vary from 0.65 at $810 \mathrm{~K}$ to 0.25 at $940 \mathrm{~K}$ indicating that the electronic and ionic conductivity are of the same order of magnitude. The drawn line will be discussed further on.

The analysis of the two-electrode impedance data allow to make a distinction between bulk and electrode contributions as can be observed in fig. 2. At high temperatures no dispersion is observed. This means that the electronic conductivity of the bulk short circuits the ionic conductivity and thus eliminates the electrode reaction. The total bulk conductivity obtained from the high frequency intercept with the real axis of the impedance spectra is represented in the Arrhenius plot of fig. 3. Comparison with literature data shows that the total conductivity of $\mathrm{BiVO}_{4}$ is appreciably lower than that of $25 \mathrm{~mol} \% \mathrm{er}$ bia-stabilized bismuth oxide (BE25) [10] and even

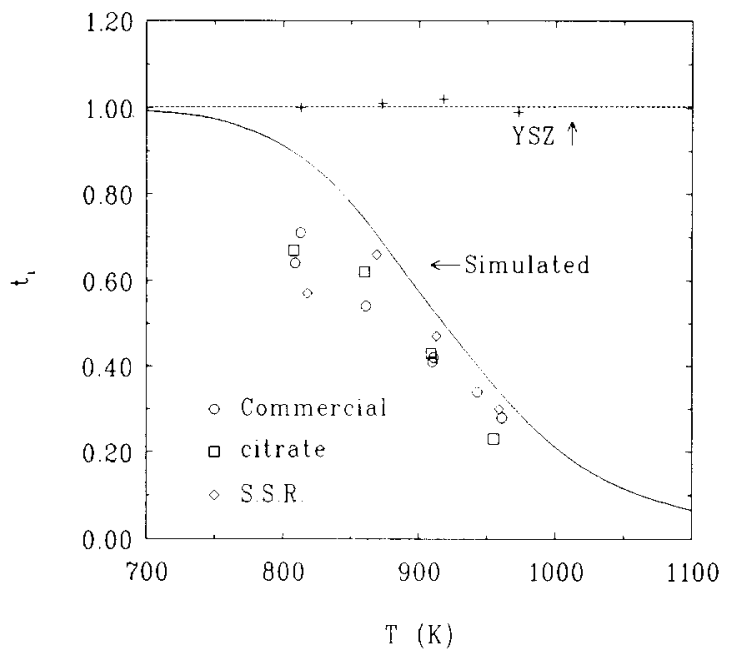

Fig. 1. Ionic transference number measurements on $\mathrm{BiVO}_{4}$ and YSZ. The drawn line represents the result from a NLLS-fit procedure.
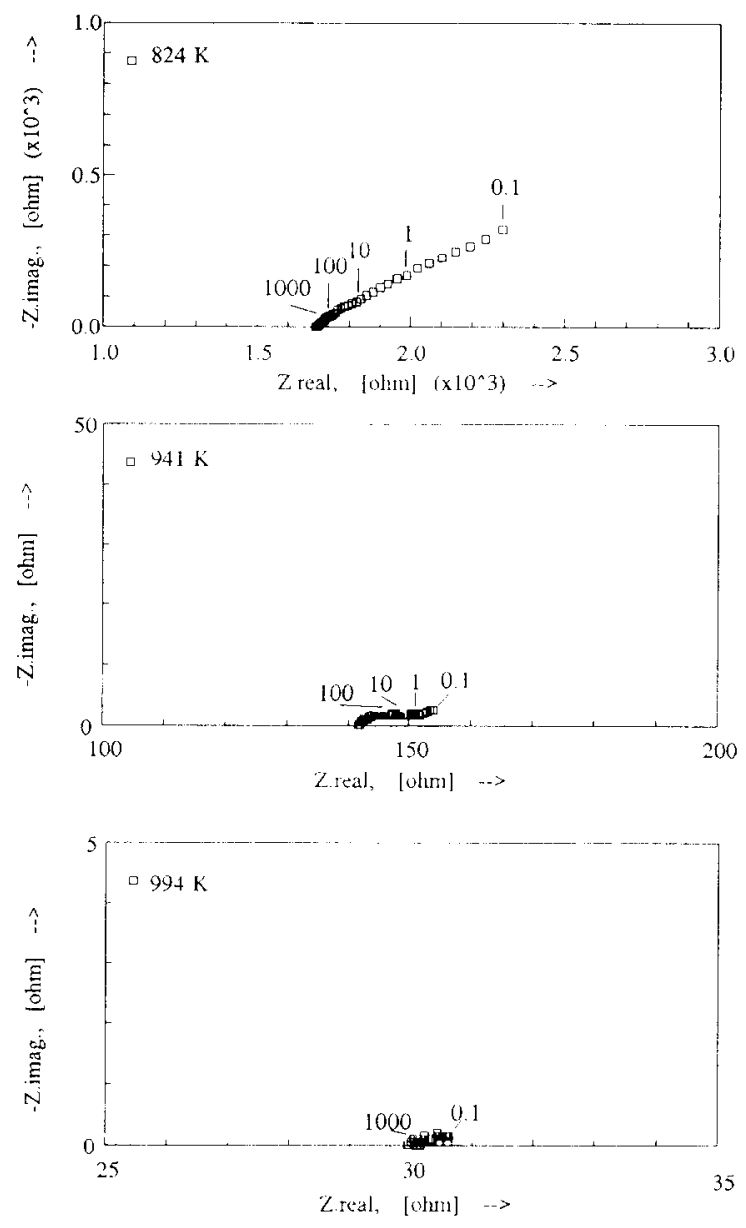

Fig. 2. Examples of frequency dispersion measurements as obtained for $\mathrm{BiVO}_{4}$ at $P_{\mathrm{O}_{3}}=8 \times 10^{-4}$ atm.

lower than the conductivity of YSZ [11]. It can be seen that at high temperatures especially the points for low $P_{\mathrm{O}_{2}}$ values deviate from a single straight line. This means that the conductivity cannot be described using a single thermally activated process. In fig. 3 we can also observe that the conductivity increases with decreasing oxygen partial pressure. This points to $n$-type electronic conductivity. To be able to distinguish between ionic and electronic conductivity we assume that the total conductivity can be described by a summation of the ionic and the electronic conductivity, see eq. (2a). Because of the indication of $n$-type mixed conductivity we assume the electronic conductivity to show Arrhenius behaviour as a function of temperature. For the $P_{\mathrm{O}_{2}}$ depend- 


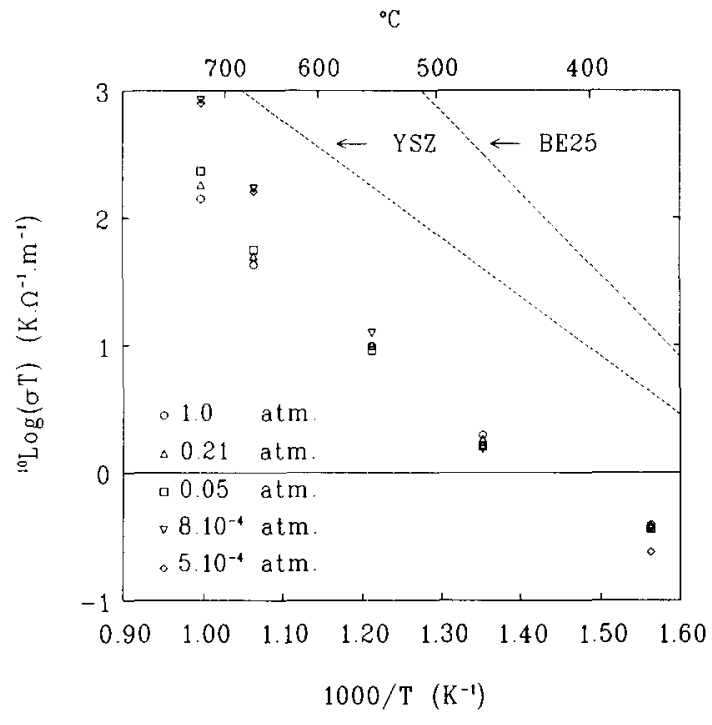

Fig. 3. Total conductivity of $\mathrm{BiVO}_{4}$ as function of temperature and oxygen partial pressure.

ency we assume that this is controlled by the reaction described by eq. (1):

$\mathrm{O}_{2, \mathrm{~g}}+2 \mathrm{~V}_{\mathrm{O}}^{\ddot{2}}+4 \mathrm{e}^{\prime} \rightleftarrows 2 \mathrm{O}_{\mathrm{O}}^{\times}$.

Assuming that the formation and mobility of the electronic charge carriers are simple thermally activated processes, eq. ( $2 \mathrm{~b}$ ) will describe the behaviour of the electronic conductivity with $n=-\frac{1}{4}$ predicted from eq. (1). As a first approximation it is assumed that the concentration of oxygen vacancies is high and is not markedly influenced by the reaction of eq. (1). Eq. (2c) describes the ionic conductivity as function of temperature and $P_{\mathrm{O}_{2}}$ :

$\sigma_{\mathrm{t}}=\sigma_{\mathrm{i}}+\sigma_{\mathrm{e}}$,

$\sigma_{\mathrm{e}}=\frac{\sigma_{\mathrm{e}, 0}}{T} \exp \left(\frac{-\Delta H}{R T}\right) P_{\mathrm{O}_{2}}^{-1 / 4}$,

$\sigma_{\mathrm{i}}=\frac{\sigma_{\mathrm{i}, 0}}{T} \exp \left(\frac{-\Delta H_{\mathrm{i}}}{R T}\right)$.

Combining the transference numbers presented in fig. 1 together with the measured total conductivities, estimates for the activation enthalpies and the pre-exponential $\sigma_{0}$ values were obtained. Using the complete set of measured impedance data (all $P_{\mathrm{O}_{2}}$ 's) these values were refined in a NLLS-fit procedure with eqs. (2a) $-(2 c)$ as model functions. Simula- tions of the electronic and ionic partial conductivities, calculated from the fit result for two $P_{\mathrm{O}_{2}}$ values, are presented in the Arrhenius plot of fig. 4 together with the experimental total conductivities. In fig. 5

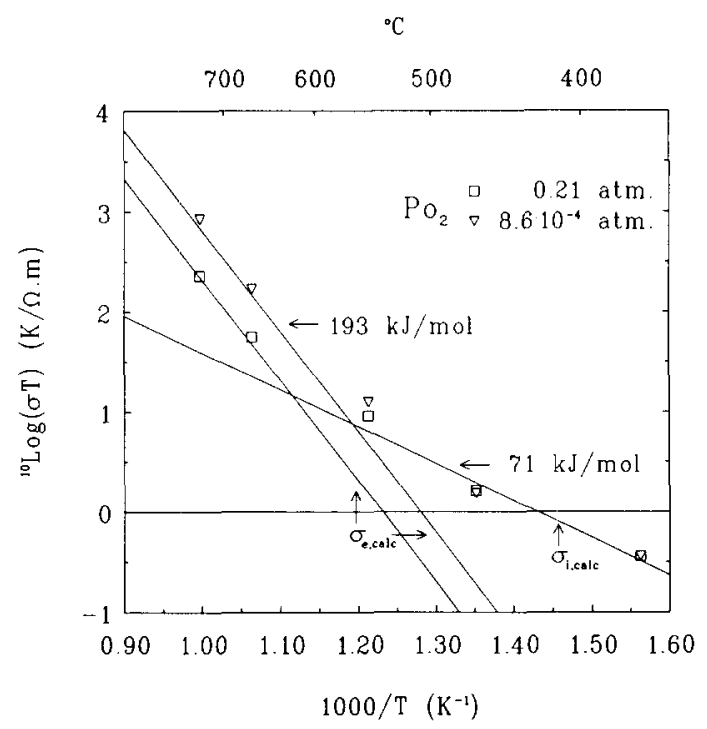

Fig. 4. Result of the NLLS-fit procedure for the ionic and electronic conductivities of $\mathrm{BiVO}_{4}$. The symbols in the graph represent the measured total conductivity. The lines represent the fit results for $P_{\mathrm{O}_{2}}=0.21 \mathrm{~atm}$ and $P_{\mathrm{O}_{2}}=8.6 \times 10^{-4} \mathrm{~atm}$.

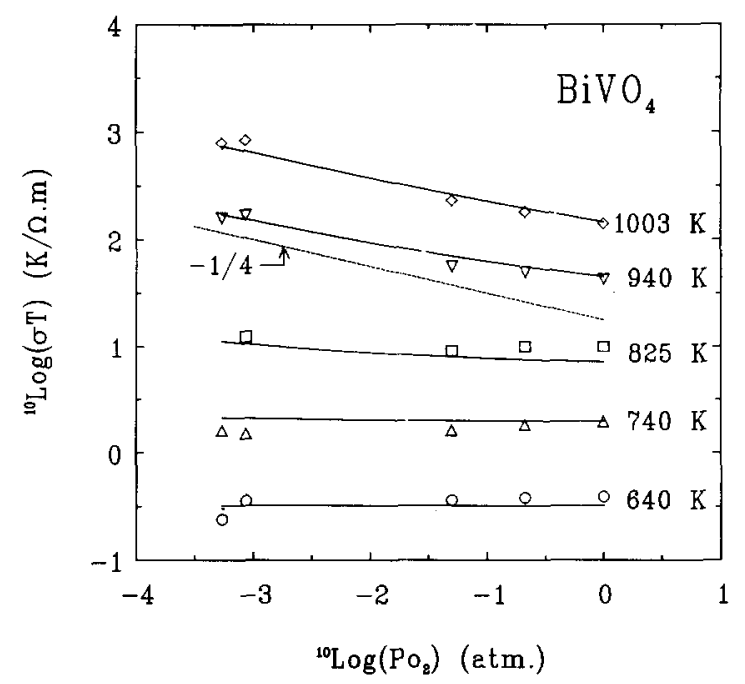

Fig. 5. $P_{\mathrm{O}_{2}}$ dependence of the total conductivity of $\mathrm{BiVO}_{4}$. The dashed line with slope $-\frac{1}{4}$ is added for comparison. 
the experimental and simulated total conductivities are presented as a function of $P_{\mathrm{O}_{2}}$ for several temperatures. This figure clearly shows that a good agreement exists between the measured and simulated values. Hence it may be assumed that the presented model based on $n$-type electronic conductivity accurately describes the temperature and $P_{\mathrm{O}_{2}}$ dependence of the measured conductivity.

A remarkable result of this analysis is the high activation enthalpy for the electronic conductivity, 193 $\mathrm{kJ} / \mathrm{mol}(2.0 \mathrm{eV})$, which is very high for an $n$-type conductor. The activation enthalpy for the ionic partial conductivity is rather low, $71 \mathrm{~kJ} / \mathrm{mol}$. As a result the total conductivity is mainly electronic at high temperatures (and low $P_{\mathrm{O}_{2}}$ values) and mainly ionic at low temperatures (and high $P_{\mathrm{O}_{2}}$ values).

Using the simulated partial electronic and ionic conductivities the ionic transference number, $t_{\mathrm{i}}=\sigma_{\mathrm{i}} /$ $\left(\sigma_{\mathrm{i}}+\sigma_{\mathrm{e}}\right)$, can be calculated for a given $P_{\mathrm{O}_{2}}$ value. The drawn line in fig. 1 represents the simulated $t_{\mathrm{i}}$ values for $P_{\mathrm{O}_{2}}=0.21 \mathrm{~atm}$. The measured $t_{\mathrm{i}}$ values are somewhat lower than the calculated values, especially at low temperatures. This difference can be attributed to the simultaneous transport of ionic and electronic species through the electrolyte (under steady state conditions) during the EMF measurement. Due to this internal oxygen transport, the oxygen activity at the electrode is not in equilibrium with the gas phase. Transference numbers calculated using the Nernst equation will therefore be too low.

Additional proof of the type of electronic charge carrier can be obtained from thermo-EMF measurements. A positive Seebeck coefficient points to positively charged majority charge carriers and a negative Seebeck coefficient to negative species. Fig. 6 shows that for $\mathrm{BiVO}_{4}$ a transition from positive to negative values can be observed in the Seebeck coefficients. This means that the majority charge carrier changes from a negatively charged species at high temperatures to a positive species at low temperatures. From the conductivity data it was concluded that at low temperatures the positively charged oxygen vacancies are the majority charge carriers. The only negative mobile species present in $\mathrm{BiVO}_{4}$ are electrons. This confirms the conclusion from the conductivity data that $\mathrm{BiVO}_{4}$ is an $n$-type mixed conductor. These results also confirm the observation that the material changes from a predominantly

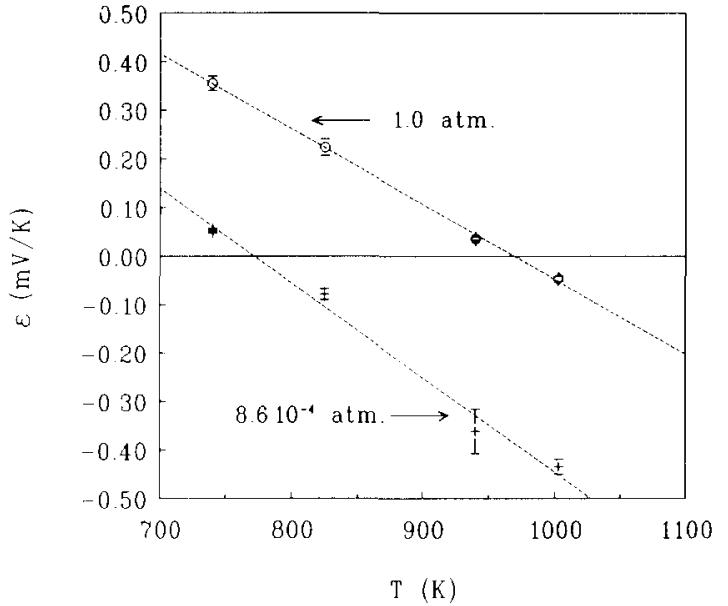

Fig. 6. Seebeck coefficients as measured for $\mathrm{BiVO}_{4}$ as function of temperature and $P_{\mathrm{O}_{2}}$.

ionic conductor at low temperatures to a predominantly electronic conductor at high temperatures.

\section{Conclusions}

Bismuth vanadate has a monoclinic cell structure.

The sintering of the material must be performed at lower temperatures to prevent closing of the skin causing low relative densities and large differences in local density of the material.

Bismuth vanadate has a total conductivity that is at least one order of magnitude lower than YSZ.

Bismuth vanadate is a mixed conductor with $n$-type electronic conductivity. The ionic and electronic conductivity are of the same order of magnitude at temperatures around $900 \mathrm{~K}$.

The activation enthalpies are $71 \mathrm{~kJ} / \mathrm{mol}$ for the ionic conductivity and $195 \mathrm{~kJ} / \mathrm{mol}$ for the electronic conductivity.

\section{Acknowledgement}

The research presented here was supported by the Netherlands Foundation for Chemical Research (SON) with financial aid from the Netherlands Organisation for Scientific Research (NWO). 


\section{References}

[1] B.C.H. Steele, J.D. Kilner, P.F. Dennis, A.E. McHale, M. van Hemert and A.J. Burggraaf, Solid State Ionics 18/19 (1986) 1038.

[2] N. Ramadass, T. Palanisamy, J. Gopalakrishnan, G. Aravamudan and M.V.C. Sastri, Solid State Commun. 17 (1975) 545.

[3] T. Lu and B.C.H. Steele, Solid State Ionics 21 (1986) 339.

[4] B.A. Boukamp, M.P. van Dijk, K.J. de Vries and A.J. Burggraaf, Adv. Ceram. 23 (1987) 445.

[5] A.J. Burggraaf, B.A. Boukamp, I.C. Vinke and K.J. de Vries, in: Advances in Solid State Chemistry, Vol, 1, ed. C.R.A. Catlow (JAI Press, London, 1989) p. 259.
[6] H. Kruidhof, K. Seshan, B.C. Lippens Jr., P.J. Gellings and A.J. Burggraaf, Mat. Res. Bull. 22 (1987) 1635.

[7] K.J. de Vries, T. van Dijk and A.J. Burggraaf, in: Fast Ion Transport in Solids, eds. P. Vashishta, J.N. Mundy and G.K. Shenoy (North-Holland, Amsterdam, 1979 ) p. 679.

[8] B.A. Boukamp, Solid State Ionics 20 (1986) 31.

[9] C. Wagner, Prog. Solid State Chem. 7 (1972) 1.

[10] M.J. Verkerk and A.J. Burggraaf, J. Electrochem. Soc. 128 (1981) 75.

[11 ] M.J. Verkerk, A.J.A. Winnubst and A.J. Burggraaf, J. Mater. Sci. 17 (1982) 3113. 